\title{
Evidence that insulin can directly inhibit hepatic glucose production
}

\author{
P.Maheux ${ }^{1}$, Y.-D.I. Chen ${ }^{1}$, K.S.Polonsky ${ }^{3}$, G.M.Reaven ${ }^{2}$ \\ ${ }^{1}$ Department of Medicine, Stanford University School of Medicine, Stanford, California, USA \\ ${ }^{2}$ Geriatric Research, Education and Clinical Center, VA Palo Alto Health Care System, Palo Alto, California, USA \\ ${ }^{3}$ Department of Medicine, Pritzker School of Medicine, University of Chicago, Chicago, Illinois, USA
}

Summary In order to evaluate the role of portal insulin in the modulation of hepatic glucose production (HGP), measurements of plasma glucose and insulin concentrations and both HGP and peripheral glucose disappearance rates were made following an infusion of a dose of tolbutamide $(0.74 \mathrm{mg}$. $\mathrm{m}^{-2} \cdot \mathrm{min}^{-1}$ ) in healthy volunteers that does not result in an increase in peripheral vein insulin concentrations or metabolic clearance rate of glucose. The results showed that the infusion of such a dose of tolbutamide was associated with a significant and rapid decline in both HGP (from $9.0 \pm 0.5$ to $7.7 \pm$ $0.5 \mu \mathrm{mol} \cdot \mathrm{kg}^{-1} \cdot \mathrm{min}^{-1}$ or $\Delta=-13.8 \pm 4.5 \% ; p<$ 0.001 compared to saline) and plasma glucose concentration (from $5.1 \pm 0.2$ to $4.4 \pm 0.1 \mathrm{mmol} / \mathrm{l}$ or $\Delta=-13.0 \pm 2.1 \% ; p<0.01$ compared to saline). Since neither HGP nor fasting glucose fell when tolbutamide-stimulated insulin secretion was inhibited by the concurrent administration of somatostatin, it indicated that tolbutamide by itself, does not directly inhibit HGP. Finally, HGP fell by $26.3 \pm 6.0 \%$ at $10 \mathrm{~min}$ after a dose of tolbutamide that elevated both peripheral and portal insulin concentrations, at a time when HGP had barely increased $(\Delta=+6.9 \pm 5.3 \%)$. The difference in the magnitude of the two responses was statistically significant $(p<0.03)$, providing further support for the view that insulin can directly inhibit HGP, independent of any change in flow of substrates from periphery to liver. [Diabetologia (1997) 40: 1300-1306]

Keywords Liver, insulin, portal system, C-peptide, tolbutamide.
Evidence has been published showing that hepatic glucose production (HGP) is decreased following the infusion of either insulin or sulfonylurea compounds [1-4], and this is generally assumed to be due to a direct effect of insulin on the liver. However, the results of two recent studies in dogs $[5,6]$ demonstrated that insulin delivered peripherally inhibited HGP as effectively as the direct intraportal administration

Received: 8 April 1997 and in revised form: 20 June 1997

Corresponding author: P. Maheux, M. D., Division of Endocrinology and Metabolism, Université de Sherbrooke, 3001 12th Avenue North, Sherbrooke, Québec, Canada JIH 5N4

Abbreviations: HGP, Hepatic glucose production; Rd, rate of disappearance or glucose uptake; MCR, metabolic clearance rate. of insulin, suggesting that the ability of insulin to inhibit HGP may be mediated through its peripheral action on substrate flow to the liver. Although we think it reasonable that insulin can inhibit HGP by decreasing substrate flow to the liver, the experiments to be presented were initiated to see whether insulin can also inhibit HGP directly. In order to accomplish this task non-invasively, we measured HGP, in normal subjects, in response to a continuous infusion of tolbutamide that would not significantly increase peripheral insulin concentrations and compared it to a saline) infusion. Under these conditions, HGP should have been similar in both studies if HGP was only modulated by the peripheral inhibition of substrate flow. Since HGP was significantly lower following tolbutamide, the data are consistent with the hypothesis that insulin can directly inhibit HGP. 


\section{Subjects and methods}

Subjects. The study population consisted of 23 healthy volunteers (13 males/10 females). All underwent a normal $75 \mathrm{~g}$ glucose tolerance test as defined by the National Diabetes Data Group [7] as well as normal routine laboratory values. The mean age was $51 \pm 12$ years, with a body mass index of $24.0 \pm 4.0 \mathrm{~kg} / \mathrm{m}^{2}$ (mean $\pm \mathrm{SD}$ ). None was taking any medication known to affect carbohydrate metabolism. This study was approved by the Stanford University Human Subjects Committee, and each individual signed a consent form upon admission to the Stanford Clinical Research Center.

Experimental protocols. Research volunteers were admitted to the Stanford Clinical Research Center on two different occasions to undergo one of three infusion protocols (Protocol 1, 2 or 3). Briefly, Protocol 1 consisted of a tolbutamide infusion at a dose that increases portal vein insulin without changing peripheral insulin concentrations. Protocol 2 consisted of infusing tolbutamide in the presence of somatostatin to verify whether tolbutamide had a direct effect on HGP. Protocol 3 involved infusion of a larger amount of tolbutamide in order to obtain a more accurate estimate of the time course of the inhibition of HGP. Details of these protocols are outlined below.

For the first two protocols, patients were admitted on two different occasions the night before each test (test A or B), and studied the following morning after a 12-h fast. At 05.30 hours, a venous catheter was inserted antecubitally in one arm and a primed $(15 \mu \mathrm{Ci}$ bolus) continuous infusion $(0.15 \mu \mathrm{Ci} / \mathrm{min})$ of HPLC purified D-[3- $\left.{ }^{3} \mathrm{H}\right]$-glucose (DupontNEN Research Products, Boston, Mass, USA) was given from 06.00 hours to 12.00 hours. We allowed $2 \mathrm{~h}$ for equilibration of the tracer ( 06.00 to 08.00 hours), and no blood samples were drawn during this period. From 08.00 hours (time $120 \mathrm{~min}$ ) onward, blood samples were obtained at 10 -min intervals for the first $60 \mathrm{~min}$, at 20 -min intervals from $180-340 \mathrm{~min}$ and again at 10 -min intervals until 360 min or 12.00 hours. Blood samples were obtained from an indwelling retrograde hand vein catheter placed in the hand contralateral to the tracer infusion site. The hand was kept in a box heated to $65^{\circ} \mathrm{F}$ for blood arterialization. Blood was collected in EDTA for measurements of plasma glucose [8], insulin [9], and C-peptide [10] concentrations.

Specific activity of $\mathrm{D}-\left[3-{ }^{3} \mathrm{H}\right]$ glucose was determined in plasma deproteinized with $\mathrm{Ba}(\mathrm{OH})_{2}$ and $\mathrm{ZnSO}_{4}[11]$. Glucose concentration was measured in the supernatant, and $\mathrm{D}\left[{ }^{3} \mathrm{H}\right]$-glucose radioactivity quantified by scintillation counting after the elimination of ${ }^{3} \mathrm{H}$-labelled water, achieved by evaporation of total water in the deproteinized plasma under compressed air. Glucose specific activity was calculated as the ratio of radioactivity to glucose in each deproteinized sample, corrected for volume dilution. Specific protocols are described as follows:

Protocol $1(n=9)$. In protocol 1, volunteers were given test A and test $B$ in random order with 3-5 days in between. In test A, a tolbutamide infusion was initiated $120 \mathrm{~min}$ after the start of the tracer infusion, and was given for $240 \mathrm{~min}(08.00$ 12.00 hours) at a continuous rate of $0.74 \mathrm{mg} \cdot \mathrm{m}^{-2} \cdot \mathrm{min}^{-1}$. This dose of tolbutamide was chosen based on preliminary experiments showing that peripheral insulin concentrations did not change in response to this infusion rate. With such an infusion rate, there was no need to infuse exogenous glucose and we could therefore assess the direct effect of portal vein insulin on HGP. Because it has been shown previously that HGP can decrease with time [12], volunteers were infused on a second occasion (Test B) with $\mathrm{D}\left[3-{ }^{3} \mathrm{H}\right]$-glucose and saline from 06.00 to 12.00 hours to obtain baseline control values.
Protocol $2(n=6)$. A second series of experiments were performed to evaluate the possibility that tolbutamide might have a direct effect on HGP, independent of insulin secretion elicited by tolbutamide. To achieve this goal, tolbutamide-induced increases in endogenous insulin secretion were blocked with somatostatin $(350 \mu \mathrm{g} / \mathrm{h})$, while basal glucagon $(0.5 \mathrm{ng}$. $\left.\mathrm{kg}^{-1} \cdot \mathrm{min}^{-1}\right)$ and insulin $\left(5 \mathrm{mU} \cdot \mathrm{m}^{-2} \cdot \mathrm{min}^{-1}\right)$ concentrations were replaced for the entire 360 -min infusion period. In Test A, tolbutamide was infused from 08.00 to 12.00 hours and measurements of glucose turnover were performed as before. Three doses of tolbutamide ( 0.74 in 1 subject; 1.45 in 2 subjects and $2.89 \mathrm{mg} \cdot \mathrm{m}^{-2} \cdot \mathrm{min}^{-1}$ in 3 subjects) were used to assess the direct effect of tolbutamide on HGP. The control study (Test B) was similar to Test A, except that saline was started at 08.00 hours. Test $\mathrm{A}$ and $\mathrm{B}$ were performed in random order.

Protocol $3(n=8)$. This last protocol was aimed at examining the time course of the inhibition of HGP with a higher dose of tolbutamide. In this protocol, volunteers were given a bolus of tolbutamide $\left(253 \mathrm{mg} / \mathrm{m}^{2}\right)$ at 08.00 hours, following $120 \mathrm{~min}$ of isotopic equilibration, followed by a 30 -min continuous infusion of tolbutamide $\left(2.89 \mathrm{mg} \cdot \mathrm{m}^{-2} \cdot \mathrm{min}^{-1}\right)$. The dose of tolbutamide was determined based on a previous publication [13], and was intended to double peripheral insulin concentrations over an infusion period of at least $30 \mathrm{~min}$. Since the infusion of exogenous glucose has been shown to inhibit HGP [14], dextrose infusion was withheld until $30 \mathrm{~min}$ after the beginning of the tolbutamide infusion. HGP and glucose disposal rate $(\mathrm{Rd})$ were calculated as in the two previous protocols. There was no control study in this particular protocol.

Calculations. Hepatic glucose production (HGP) and Rd were calculated using Steele's non-steady-state equation [15] as modified by De Bodo [16]. This equation is derived from a one-compartment model and the fixed pool fraction used was 0.65 . Before being used in the Steele equation, values of $\mathrm{D}[3$ $\left.{ }^{3} \mathrm{H}\right]$-glucose specific activities, as well as plasma glucose levels, were smoothed using the optimal segment method [17]. In protocol 1, 2 and 3, HGP are plotted as values relative to baseline: the latter defined as the average of the three 10-min intervals of the last $30 \mathrm{~min}$ of the isotopic equilibration period (90 to $120 \mathrm{~min}$ ). Absolute values were also analysed and are reported in the Results Section.

Statistical analysis. Data are expressed as the mean \pm SEM and were analysed by the Statistical Analysis System (SAS) program (SAS Inc., Cary, N.C., USA), using the general linear model procedure. Comparisons between the tolbutamide and its control infusion were performed using a repeated measure of two-factor analysis of variance (time $\times$ treatment group) within each subject. When indicated, a one-way analysis of variance (ANOVA) was also used to compare time courses of glucose, insulin, or glucose turnover data for a given infusion within subjects. In addition, paired and unpaired Student's $t$ tests were used in Protocols 1, 2 and 3 to compare some specific aspects in the kinetics of glucose, insulin, HGP and Rd.

\section{Results}

Protocol 1. Figure 1 compares the effects of tolbutamide or saline on changes in plasma insulin, C-peptide and glucose concentrations. As expected, the data in the top panel show that peripheral plasma insulin concentrations were identical throughout the entire $240 \mathrm{~min}$ following the infusion of either the 
lower dose of tolbutamide $\left(0.74 \mathrm{mg} \cdot \mathrm{m}^{-2} \cdot \mathrm{min}^{-1}\right)$ or saline. However, despite identical peripheral plasma insulin concentrations, the results in the middle panel of Figure 1 demonstrate that concentrations of Cpeptide were significantly higher throughout the 240-min infusion period in response to the infusion of tolbutamide $(p<0.001$ using a two-factor ANOVA). More specifically, C-peptide increased from a baseline value of $0.38 \pm 0.05 \mathrm{nmol} / 1$ to a maximum of $0.44 \pm 0.06 \mathrm{nmol} / 1 \quad(\Delta=+15.5 \pm 2.5 \% ; p<0.01$ by paired $t$-test) $40 \mathrm{~min}$ after the beginning of the tolbutamide infusion. In contrast, $\mathrm{C}$-peptide concentrations during the saline infusion fell from $0.39 \pm$ $0.04 \mathrm{nmol} / \mathrm{l}$ at $120 \mathrm{~min}$ to $0.35 \pm 0.03 \mathrm{nmol} / \mathrm{l}$ at $160 \min (\Delta=-9.3 \pm 4.7 \% ; p>0.05)$ and even lower to $0.29 \pm 0.04 \mathrm{nmol} / 1$ at $360 \mathrm{~min}$. Finally, the lower panel of Figure 1 depicts the plasma glucose concentrations resulting from the infusion of tolbutamide $\left(0.74 \mathrm{mg} \cdot \mathrm{m}^{-2} \cdot \mathrm{min}^{-1}\right)$ as compared to the saline control. It can be seen that plasma glucose concentrations decreased to a significantly greater degree in response to the tolbutamide infusion (from $5.1 \pm 0.2$ to $4.4 \pm 0.1 \mathrm{mmol} / 1$ or $\Delta=-13.0 \pm 2.1 \%$ vs $\Delta=-2.8 \pm$ $2.2 \%$ with the saline infusion; $p<0.01$ ).

Figure 2 illustrates the relative changes in $\mathrm{Rd}$ (top panel) and HGP (bottom panels) rates during the two infusion studies. Without any changes in peripheral insulin concentrations, it can be seen that $\mathrm{Rd}$ did not change substantially in response to the tolbutamide or the saline infusion (Fig.2, top panel; $p>$ 0.05 ). In contrast, HGP decreased in absolute values from an average of $9.0 \pm 0.5$ to $7.7 \pm 0.5 \mu \mathrm{mol} \cdot \mathrm{kg}^{-1}$. $\min ^{-1}$ (Fig. 2, middle panel) and this difference was statistically significant when compared to the saline infusion by two-way ANOVA $(p<0.001)$. When HGP data were plotted relative to baseline values (a mean of HGP in the last 30 min before the infusion), the difference between the saline and tolbutamide infusions was obvious and remained statistically significant ( $p<0.001$ using a two-way ANOVA over the entire time course; Fig. 2, lower panel). It is worth noting that the suppression of HGP occurred within $10 \mathrm{~min}$ of the tolbutamide infusion protocol $(\Delta=$ $-9.9 \pm 5.9 \%$ ) and remained so for a significant portion of the study.

To present these data from a different perspective, the comparative effects on Rd and HGP in response to the saline (top panel) and tolbutamide (middle panel) infusions are illustrated in Figure 3. It is apparent that Rd and HGP were essentially identical during the saline infusion (Fig. 3, top panel), consistent with the finding that plasma glucose did not change during the saline infusion (Fig. 1, lower panel). In contrast, HGP decreased in response to tolbutamide ( $p<0.001$; Fig. 3, middle panel), accounting for the significant decline in plasma glucose concentration observed in response to the tolbutamide infusion (Fig. 1, lower panel). To assess whether a peripheral
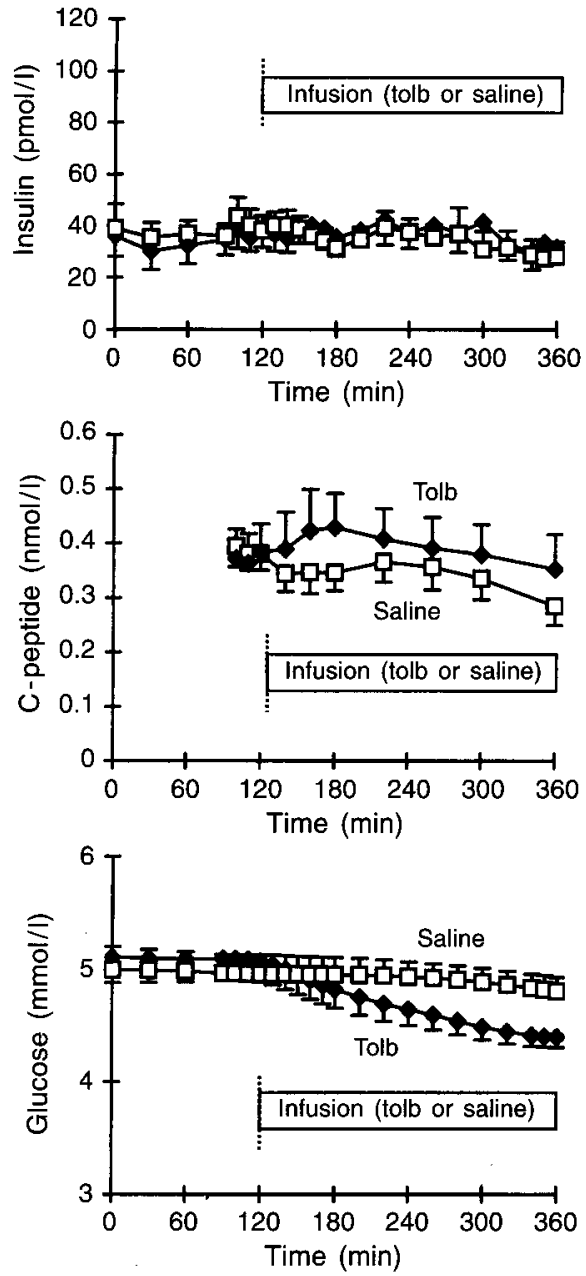

Fig. 1. Comparison of effects of tolbutamide $\left(\diamond, 0.74 \mathrm{mg} \cdot \mathrm{m}^{-2}\right.$. $\mathrm{min}^{-1}$ ) vs saline $(\square)$ on plasma insulin (top panel), C-peptide (middle panel) and glucose concentrations (lower panel)

effect of insulin had occurred, we plotted the metabolic clearance rate (MCR) of glucose (Rd divided by the ambient plasma glucose) over the entire duration of the tolbutamide and the saline infusion. It is obvious from Figure 3 (lower panel) that the MCR of glucose was not changed by the infusion of a small dose of tolbutamide $(p=0.59$ using a two-factor ANOVA).

Protocol 2. The data in Figure 4 show the results of experiments in which the effects of tolbutamide and saline on plasma insulin, glucose, and HGP were compared in the presence of a continuous infusion of somatostatin (to suppress the endogenous insulin secretion). Data are expressed as pooled values for the three different doses of tolbutamide and the statistical analysis was performed using paired values for each individual. It is apparent that plasma insulin and glucose concentrations and HGP were essentially identical during the infusion of tolbutamide or saline $(p>0.05)$. It should be appreciated that exogenous insulin and glucagon were also infused during these 

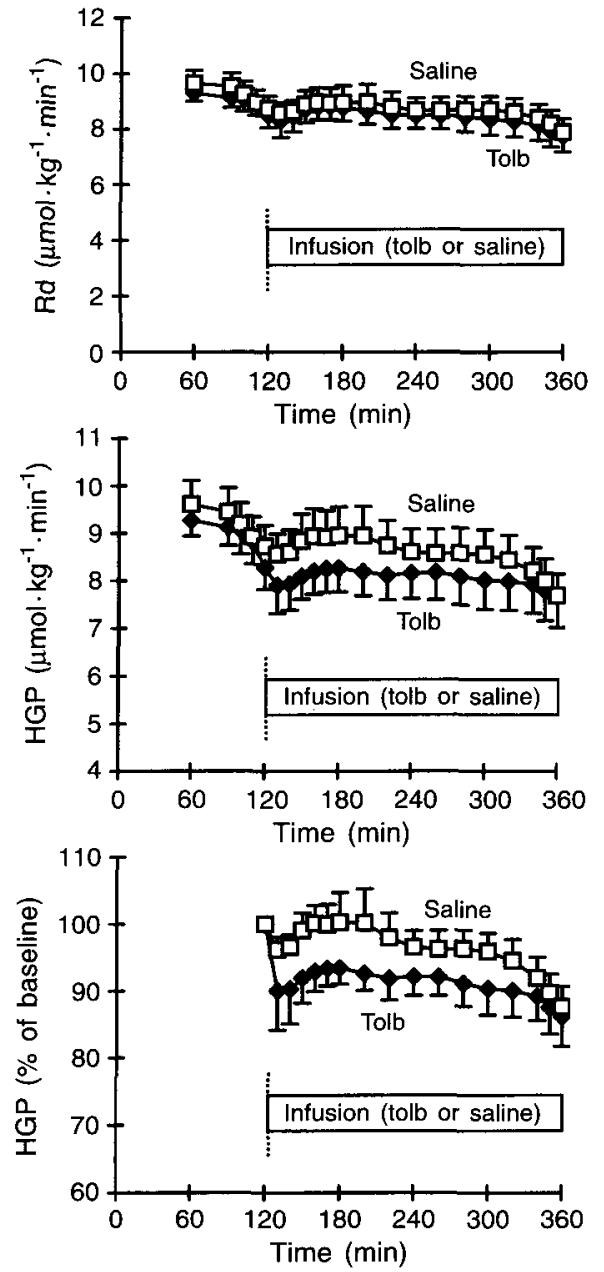

Fig. 2. Comparison of effects of tolbutamide $\left(\diamond, 0.74 \mathrm{mg} \cdot \mathrm{m}^{-2}\right.$. $\mathrm{min}^{-1}$ ) vs saline control ( $\square$ ) on glucose disposal (Rd - top panel) and hepatic glucose production in absolute (HGP - middle panel) or relative values to baseline (HGP - lower panel)

studies in order to maintain basal concentrations of both hormones during the experiments.

Protocol 3. Figure 5 shows the effects- of a primedcontinuous infusion of tolbutamide $\left(253 \mathrm{mg} / \mathrm{m}^{2}\right.$ followed by $2.89 \mathrm{mg} \cdot \mathrm{m}^{-2} \cdot \mathrm{min}^{-1}$ for $30 \mathrm{~min}$ ) on changes in plasma insulin and glucose concentrations, $\mathrm{Rd}$ and HGP. In response to this infusion of tolbutamide, the results in the top panel show that there was a brisk increase in plasma insulin concentration during the first $10 \mathrm{~min}$ from $39 \pm 6$ to $152 \pm 38 \mathrm{pmol} / \mathrm{l}(p<0.001)$, followed by a progressive decline. Plasma glucose concentrations fell progressively as seen in the middle panel, reaching $80.7 \pm 2.2 \%$ of the baseline value or $3.9 \pm 0.1 \mathrm{mmol} / 1$ at $30 \mathrm{~min}(p<0.001$ by one-way ANOVA). The lower panel of Figure 5 illustrates the changes in Rd and HGP rates during this high dose tolbutamide infusion study. It can be seen that Rd increased to $121.0 \pm 9.1 \%$ of the baseline value $(\Delta=$ $+21.0 \pm 9.1 \%$ ) during the first $30 \mathrm{~min}$ of the tolbutamide infusion while the decrease in HGP tended to
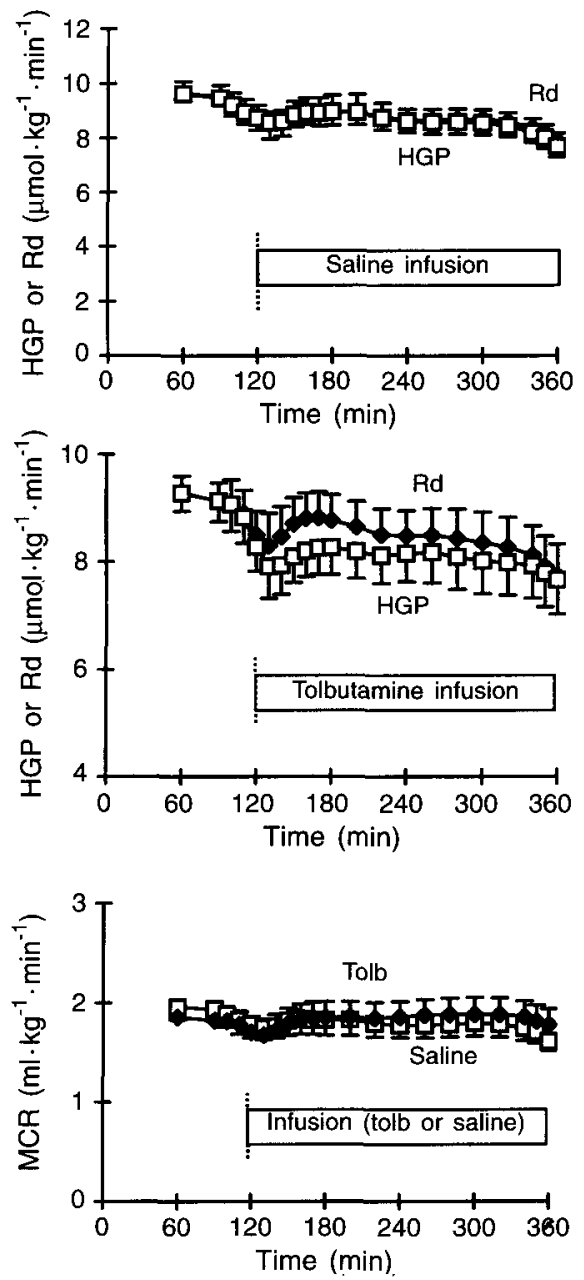

Fig. 3. Comparison of glucose disposal ( $\mathrm{Rd}, \bullet)$ and hepatic glucose production (HGP, $\square$ ) rates in response to infusion of either saline (top panel) or tolbutamide $\left(0.74 \mathrm{mg} \cdot \mathrm{m}^{-2} \cdot \mathrm{min}^{-1}\right.$ - middle panel). MCR of glucose is compared in the bottom panel between low dose tolbutamide infusion $(\bullet, 0.74 \mathrm{mg}$. $\mathrm{m}^{-2} \cdot \min ^{-1}$ ) and saline control $(\square)$

be more important $(\Delta=-32.8 \pm 7.0 \%$ for HGP vs $\Delta=+21.0 \pm 9.0 \%$ for $\mathrm{Rd} ; p>0.05$ by unpaired $t$ test). More importantly, the inhibition of HGP was very rapid, and within $10 \mathrm{~min}$ after the start of the tolbutamide infusion, HGP had fallen by $26.3 \pm$ $6.0 \%$. The fall in HGP $(\Delta=-26.3 \pm 6.0 \%)$ occurred before and was of greater magnitude than either the increase in $\operatorname{Rd}(\Delta=+6.9 \pm 5.3 \% ; p<0.03$ by $t$-test $)$ or the fall in plasma glucose $(\Delta=-7.3 \pm 0.8 \% ; p<$ 0.001 by $t$-test).

\section{Discussion}

The goal of these experiments was to see whether an increase in portal vein insulin concentration could inhibit HGP, independent of an increase in peripheral plasma insulin concentration. In order to accomplish this task, we used an experimental approach based 

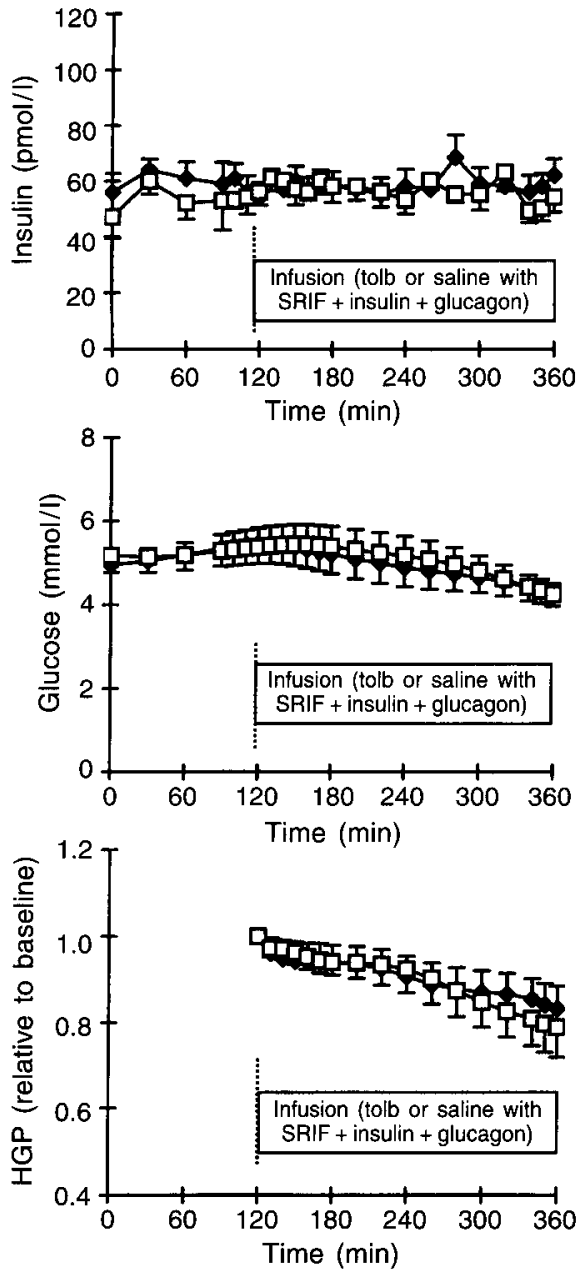

Fig.4. Comparison of effects of tolbutamide ( $\$, 0.74$ $2.89 \mathrm{mg} \cdot \mathrm{m}^{-2} \cdot \mathrm{min}^{-1}$ ) vs saline ( $\square$ ) on insulin (top panel), glucose (middle panel) and hepatic glucose production (HGP lower panel) in the presence of somatostatin plus glucagon and insulin replacement

on the premise that portal insulin concentration would always be higher than peripheral insulin concentration during an infusion of tolbutamide. Although this assumption was based upon the generally accepted belief that tolbutamide directly stimulates pancreatic insulin secretion, direct evidence in support of the view has recently been published [18]. Therefore, if similar peripheral insulin concentrations are seen following the tolbutamide and saline infusions, HGP should be lower following tolbutamide if increases in portal insulin directly inhibit HGP. The results presented in Figures $1-3$ provide direct evidence in support of this formulation. Thus, the data shown in the lower panels of Figures 1 and 2 demonstrate that both plasma glucose concentration and HGP were significantly lower during the 240-min infusion of tolbutamide compared to the saline control. This was seen despite the fact that peripheral insulin levels were similar during the infusion of the two test substances (Fig. 1 top). When the
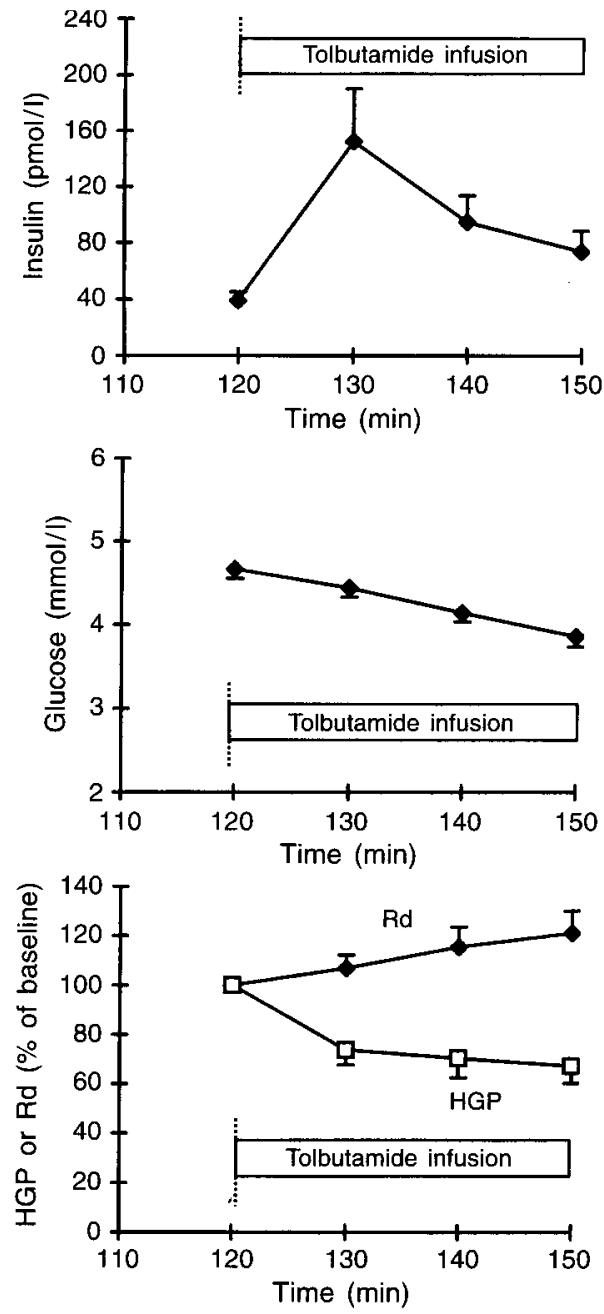

Fig. 5. Effects of a high tolbutamide dose $\left(253 \mathrm{mg} / \mathrm{m}^{2}+\right.$ $2.89 \mathrm{mg} \cdot \mathrm{m}^{-2} \cdot \mathrm{min}^{-1}$ ) on plasma insulin (top panel) and glucose (middle panel) concentrations, as well as on glucose turnover data (Rd, $\diamond$ and HGP, $\square-$ lower panel)

effect of tolbutamide on regulation of Rd and HGP was compared to that of the saline control infusion (Fig. 3), it can be seen that although both variables changed throughout the study there was a significant decrease in HGP, relative to the changes in Rd in responses to the tolbutamide, as compared to the saline infusion. Since C-peptide levels were significantly higher in response to the tolbutamide infusion (Fig.1), it seems almost certain that portal vein insulin concentrations were also increased, despite the absence of peripheral hyperinsulinaemia. Thus, the fall in plasma glucose and HGP (Figs. 1 and 2) strongly suggests that increased levels of insulin in the portal vein can inhibit HGP independently of peripheral hyperinsulinaemia. On the other hand, we cannot rule out the possibility that a small increase in peripheral insulin level, not detected by our assay, could have inhibited lipolysis, and the subsequent decline in NEFA level could have contributed to the fall in HGP. However, the absence of an increase in 
the MCR of glucose (Fig. 3, lower panel) in response to tolbutamide as compared to saline suggests that a peripheral effect of insulin was not present. Indeed, in protocol $1, \mathrm{HGP}$ and $\mathrm{Rd}$ moved at the same rate during the tolbutamide infusion, resulting in a glucose MCR for glucose that was virtually flat during the infusion of either saline or tolbutamide. These calculations provide some support for the fact that the fall in HGP was independent of a significant peripheral effect of insulin.

The experiment outlined in Protocol 2 were performed in order to rule out the possibility that the fall in HGP seen during tolbutamide administration in Protocol 1 was not due to a direct effect of tolbutamide to inhibit HGP. The finding (Fig. 4) that neither plasma glucose concentration nor HGP decreased when tolbutamide was given in association with somatostatin demonstrates that tolbutamide per se does not directly inhibit HGP. However, it could be argued that tolbutamide could indirectly affect HGP by suppressing glucagon levels $[19,20]$. In previous studies, this suppressive effect of tolbutamide on glucagon release was seen at tolbutamide infusion rates 5-15 times greater than those used in the current study, and only occurred when plasma glucose concentrations were clamped in response to tolbutamide [20]. However, when plasma glucose concentrations were allowed to fall, as in the lower panel of Figure 1 in the current study, there was absolutely no discernible change in glucagon concentrations [19]. Furthermore, it also appears from results of recent studies that sulfonylureas do not influence alpha-cell function, either directly or indirectly by a paracrine effect via insulin suppression [21]. While glucagon concentrations were not measured in the present study, it is unlikely that such a small dose of tolbutamide could have had any significant impact.

Finally, the glucose turnover data (HGP and Rd) in response to a primed-continuous and higher dose of tolbutamide in Protocol 3 provide supplemental evidence that insulin can inhibit HGP directly. In these studies, a higher dose of tolbutamide increased portal and peripheral insulin concentrations, leading to an increase in $\mathrm{Rd}(\Delta=+21.0 \pm 9.1 \%)$ and a decrease in HGP $(\Delta=-32.8 \pm 7.0 \%)$ at $30 \mathrm{~min}$. Within 10 min of the beginning of the tolbutamide infusion, there was a $26.3 \pm 6.0 \%$ drop in HGP as compared to a $6.9 \pm 5.3 \%$ rise in $\mathrm{Rd}$, and the magnitude of this difference was statistically significant $(p<0.03)$. These data are reminiscent of earlier works from Prager et al. [22] demonstrating that the inhibition of HGP was more rapid than the increase in $\mathrm{Rd}$. A few years later, Bergman et al. [17, 23, 24] showed that Rd was a "sluggish process", dependent on a transcapillary transport of insulin at the level of the skeletal muscle. In these experiments, Bergman demonstrated that half the stimulation of $\mathrm{Rd}$, in the context of a hyperinsulinaemic clamp, was obtained 4045 min after the administration of insulin, and that it was highly dependent on the increase in lymph (interstitial) insulin concentrations. If one assumes that a small dose of tolbutamide (such as the one used in Protocol 1), that did not lead to a measurable increase in plasma insulin can affect substrate flow from periphery to liver within $10 \mathrm{~min}$, it implies a minimal role of this postulated rate-limiting step of insulin transport from plasma to tissue. Finally, in addition to the absence of any measurable increase in plasma insulin concentrations, and the rapidity of the decrease in HGP, there is the fact that MCR of glucose did not change in our low-dose tolbutamide protocol. All of these considerations support the notion that insulin directly inhibit HGP.

The results presented in this study have shown that increases in portal vein insulin concentration can inhibit HGP in the absence of increases in peripheral vein insulin concentration. As such, they support the view that insulin can directly inhibit HGP. This conclusion should not be construed to imply that insulin per se is the major regulator of HGP. For example, there are two publications $[5,6]$ showing that the direct administration of insulin into the portal vein is no more powerful than peripherally administered insulin in suppression of HGP when peripheral insulin concentrations are perfectly matched. On the other hand, interpretation of these studies is confounded by the fact that glucose had to be infused in significant amounts in order to maintain euglycaemia when the insulin was administered peripherally and we have previously shown the powerful inhibitory effect of a glucose infusion on HGP, in the absence of any apparent change in plasma glucose concentration [14].

The current results do not permit us to make any quantitative estimates of the relative importance of a direct effect of insulin on inhibition of HGP, as compared to an indirect effect by decreasing substrate flow to the liver. For example, the C-peptide levels increased to a greater degree during the tolbutamide infusion than was the associated decrease in either plasma glucose concentration or HGP. Thus, it could be argued that the ability of insulin to directly inhibit HGP may be relatively small in magnitude. Indeed, this conclusion is quite consistent with evidence that inhibition of HGP by insulin occurs both directly, as well as indirectly by inhibiting substrate flow from peripheral tissues to the liver $[20]$ ]' In the present study, it is unlikely that the infusion of a minute dose of tolbutamide (insufficient to raise peripheral insulin levels), could have had a peripheral effect on substrate flow. Therefore, these arguments point to a direct action of portal vein insulin on HGP.

In conclusion, the results presented have shown that tolbutamide can inhibit HGP and lower plasma 
glucose concentration in the absence of any increase in peripheral plasma insulin concentration. Furthermore, this effect of tolbutamide on HGP and plasma glucose can be seen in the absence of any increase in peripheral glucose disposal rate. As such, these data provide direct evidence that portal insulin can act directly on the liver to inhibit HGP.

Acknowledgements. We wish to acknowledge Mr. John L. Jacobs and Mr. Robert A. Parillo as well as the Upjohn Company for their generous contribution in providing tolbutamide for these studies. Work was supported by grants from the National Institute of Health (DK30732, RR00070), the Diabetes Research and Training Center at The University of Chicago (DK20595) and the Endocrine Fellows Foundation (New York). Dr. Maheux was supported by a Fellowship from the Medical Research Council of Canada.

\section{References}

1. Steele R, Bishop JS, Dunn A, Altszuler N, Rathgeb I, De Bodo RC (1965) Inhibition by insulin of hepatic glucose production in the normal dog. Am J Physiol 208: 301-306

2. Chiasson JL, Liljenquist JE, Finger FE, Lacy WW (1976) Differential sensitivity of glycogenolysis and gluconeogenesis to insulin infusions in dogs. Diabetes 25: 283-291

3. Groop L, Luzi L, Melander A et al. (1987) Different effects of glyburide and glipizide on insulin secretion and hepatic glucose production in normal and NIDDM subjects. Diabetes 36: $1320-1328$

4. Rizza RA, Mandarino LJ, Gerich JE (1981) Dose-response characteristics for effects of insulin on production and utilization of glucose in man. Am J Physiol 240: E630-E639

5. Ader M, Bergman RN (1990) Peripheral effects of insulin dominate suppression of fasting hepatic glucose production. Am J Physiol 258: E1020-E1032

6. Giacca A, Fisher SJ, Shi ZQ, Gupta R, Lickley HL, Vranic $M$ (1992) Importance of peripheral insulin levels for insulin-induced suppression of glucose production in depancreatized dogs. J Clin Invest 90: 1769-1777

7. National Diabetes Data Group (1979) Classification and diagnosis of diabetes mellitus and other categories of glucose intolerance. Diabetes 28: 1039-1057

8. Kadish AH, Litle RL, Sternberg JC (1968) A new and rapid method for the determination of glucose by measurement of rate of oxygen consumption. Clin Chem 14: 116131

9. Hales CN, Randle PJ (1963) Immunoassay of insulin with insulin-antibody precipitate. Biochem J 88: 137-146
10. Faber OK, Binder C, Markussen J et al. (1978) Characterization of seven C-peptide antisera. Diabetes 27 [Suppl 1]: $170-177$

11. Somogyi M (1945) Determination of blood sugar. J Biol Chem 10: 69-73

12. Chen Y-Di, Swislocki AL, Jeng C-Y, Juang JH, Reaven GM (1988) Effect of time or measurement of hepatic glucose production. J Clin Endocrinol Metab 67: 1084-1088

13. Ganda OP, Kahn CB, Soeldner JS, Gleason RE (1975) Dynamics of tolbutamide, glucose, and insulin interrelationships following varying doses of intravenous tolbutamide in normal subjects. Diabetes 24: 354-367

14. Sheu WH-H, Hollenbeck CB, Wu M-S, Jaspan JB, Chen YDI, Reaven GM (1990) Effect of difference in glucose infusion rate on quantification of hepatic glucose production. $\mathrm{J}$ Clin Endocrinol Metab 70: 1354-1360

15. Steele R (1959) Influences of glucose loading and of injected insulin on hepatic glucose output. Ann NY Acad Sci USA 82: $420-430$

16. De Bodo RC, Steele R, Altszuler N, Dunn A, Bishop JS (1963) On the hormonal regulation of carbohydrate metabolism: studies with C-14 glucose. Rec Prog Horm Res 19: 445-448

17. Bradley DC, Poulin RA, Bergman RN (1993) Dynamics of hepatic and peripheral insulin effects suggest common ratelimiting step in vivo. Diabetes 42: 296-306

18. Lewis GF, Steiner G, Polonsky KS, Weller B, Zinman B (1994) A new method for comparing portal and peripheral venous insulin delivery in humans: tolbutamide vs insulin infusion. J Clin Endocrinol Metab 79: 66-70

19. Pfeifer MA, Beard JC, Halter JB, Judzewitsch R, Best JD, Porte Jr D (1983) Suppression of glucagon secretion during a tolbutamide infusion in normal and noninsulin-dependent diabetic subjects. J Clin Endocrinol Metab 56: 586-591

20. Lewis GF, Zinman B, Groenewoud Y, Vranic M, Giacca A (1996) Hepatic glucose production is regulated both by direct and extrahepatic effects of insulin in humans. Diabetes 45: $454-462$

21. Gregorio F, Ambrosi F, Cristallini S, Pedetti M, Filipponi P, Santeusanio F (1992) Therapeutic concentrations of tolbutamide, glibenclamide, gliclazide and gliquidone at different glucose levels: in vitro effects on pancreatic A- and B-cell function. Diabetes Res Clin Pract 18: 197-206

22. Prager R, Wallace P, Olefsky JM (1987) Direct and indirect effects of insulin to inhibit hepatic glucose output in obese subjects. Diabetes 36: 607-611

23. Steil GM, Meador MA, Bergman RN (1993) Thoracic duct lymph: relative contribution from splanchnic and muscle tissue. Diabetes 42: 720-731

24. Ader M, Bergman RN (1994) Importance of transcapillary insulin transport to dynamics of insulin action after intravenous glucose. Am J Physiol 266: E17-E25 